\title{
Eleven-year surveillance of acute flaccid paralysis in southwestern Iran
}

\author{
Ali Akbar Momen ${ }^{1}$, Abdolhussein Shakurnia ${ }^{2}$, Mehdi Momen ${ }^{3}$ \\ Departments of ${ }^{1}$ Pediatric and ${ }^{2} I m m u n o l o g y$, School of Medicine, Ahvaz Jundishapur University of Medical Sciences, \\ Ahvaz, Iran; ${ }^{3}$ Internal Medicine Resident at Elmhurst Hospital, NY, USA. E-mail: shakurnia@yahoo.com \\ Received: 27th November 2017, Accepted: 31st October 2018
}

SUMMARY: Momen AA, Shakurnia A, Momen M. Eleven-year surveillance of acute flaccid paralysis in southwestern Iran. Turk J Pediatr 2019; 61: 544-551.

The purpose of the study was to report the results of acute flaccid paralysis (AFP) surveillance in the southwestern Iran in the 11 years' period. This is a retrospective descriptive study based on WHO guidelines. The collected data includes all pediatric AFP cases reported between of January 2006 to December 2016 by all health centers of Khuzestan province in Southwest of Iran. During an eleven-year period, 274 cases of AFP were reported in Khuzestan Province. Among them, 56.9\% were younger than five years of age, and $55.9 \%$ were male. None of these cases was confirmed as poliomyelitis. The annual incidence of non-polio AFP ranged from 1.46/100,000 to 3.11/100,000 (Mean: 2.04 cases/100,000). The incidence rate of AFP in children under five years of age was significantly higher compared to older children $(p=0.001)$, and the most common cause of paralysis $(74.5 \%)$ was Guillain-Barre syndrome. All performance indicators met the WHO-specified targets. The follow-up of patients with AFP is essential to the eradication of poliomyelitis.

Key words: acute flaccid paralysis, surveillance, poliomyelitis eradication, southwestern Iran.

Poliomyelitis is a highly contagious viral disease spreading through human-to-human contact. ${ }^{1}$ A global effort began by the World Health Organization (WHO) as of 1988 to eradicate poliomyelitis, which adopted the Global Polio Eradication Initiative (GPEI) worldwide. ${ }^{2}$ These efforts have reduced the annual incidence of polio and have led to the official eradication of it in most countries. Immunization and surveillance are the mainstays of this WHO-led strategy. The GPEI program involves the implementation of an Acute Flaccid Paralysis (AFP) surveillance system with the purpose of detecting the wild origin of poliovirus isolates and achieving a high rate of polio immunization coverage in response to polio outbreaks. All countries are encouraged to implement a surveillance system with the goal of detecting AFP. ${ }^{3-5}$

Acute Flaccid Paralysis is part of the clinical presentation of poliomyelitis, which makes the AFP surveillance system a critical tool in detecting poliomyelitis among children under the age of fifteen. AFP surveillance system, which is a golden strategy in polio eradication that creates an important responsibility for public health systems in most countries, has been globally recommended as a key strategy to monitor the progress of the polio eradication initiative. This program can be helpful in identifying high-risk countries or populations to monitor polio status in each geographical region. ${ }^{6,7}$

In line with the goals of WHO, a robust surveillance system for AFP reporting has been established in Iran since $1990 .^{8}$ During recent decades, Iran has achieved successes in the control and eradication of infectious diseases. The existence of an excellent and easily accessible primary health care system in rural and urban health centers is a cornerstone to achieving polio eradication in Iran. ${ }^{9}$ The regular use of Oral Polio Vaccine (OPV) in Iran, the high coverage of routine immunization 
in six doses of OPV3 during six years after birth as well as the existence of a sensitive surveillance system have led to the eradication of polio in this country. The routine OPV 3 administration has achieved a coverage of nearly $100 \%$ since $2002^{10}$. The last case of wild poliovirus (imported from Afghanistan) was detected in December 2000 in the country, which was confirmed in 2001 by WHO, and Iran has been currently been identified as a polio-free country. ${ }^{11,12}$

Acute flaccid paralysis is defined as the acute or sudden onset of paralysis or weakness in one or more limbs, respiratory muscles or bulbar muscles resulting from damaged to the lower motor neurons. Poliomyelitis was the most important cause of AFP, but since its decline, other causes have emerged. AFP is diagnosed clinically by taking a thorough medical history and physical examination. It is a clinical syndrome caused by a wide range of probable etiologies, including Guillain-Barre syndrome (GBS), non-polio Enteroviruses, poliomyelitis, toxins, and acute traumatic neuritis. ${ }^{13,14}$

AFP surveillance program mandates physicians to report any case of AFP in children under the age of fifteen to the health authorities using AFP surveillance guidelines. WHO has developed indicators that determine the quality of the AFP surveillance system. In a non-endemic country such as Iran, the incidence of at least one case of non-polio AFP per 100,000 children under the age of fifteen is expected. ${ }^{15,16}$

During the past few years, Poliovirus transmission has been reported at its lowest levels; however, the virus continues to spread from endemic countries, and the high level of immigration across countries creates an environment for Polio Virus reintroduction. ${ }^{17}$ Despite the overall success of GPEI in Iran, as well as the absence of confirmed polio cases in the country since $2001^{11}$, Iranian authorities remain concerned regarding the possibility of transmission from two polio-endemic neighboring countries of Afghanistan and Pakistan, which are both at a high risk of wild poliovirus. ${ }^{18}$ The nationwide surveillance of AFP and environmental control in combination with extensive polio vaccination are critical considering the presence of wild poliovirus subtypes around the world.

Poliomyelitis is on the verge of elimination in the world, which makes other causes of AFP in children more prominent. Given that WHO is running a polio eradication campaign, many studies are significant from an epidemiological standpoint. Several studies have been conducted on AFP cases and the efficacy of the surveillance system in different countries around the globe. ${ }^{15}$ Since diagnosing and identifying AFP cases in every country is a part of the surveillance system for the eradication of poliomyelitis, assessing poliovirus surveillance efficacy in different regions is essential to support the global disease eradication efforts. This study was conducted to determine the epidemiological and geographic distribution of AFP cases in Khuzestan Province of Southwestern Iran from January 2006 to December 2016.

\section{Material and Methods}

In this retrospective study, we analyzed all children under the age of fifteen presenting with AFP from January 2006 to December 2016. This case series study has been developed by the expert AFP committee of Ahvaz Jundishapur University of Medical Sciences (AJUMS) in Khuzestan Province with the contribution of vice-chancellor of health. Khuzestan Province is located on the southwest border of Iran and has a hot and dry climate. The population of the province is more than 4,700,000 with approximately $1,200,000$ individuals under the age of fifteen. ${ }^{19}$

Ethical approval was granted by AJUMS Research and Ethics Committee. The cases were reviewed using the results of a surveillance system for non-polio AFP, which was conducted according to the WHO recommendations for global eradication of poliomyelitis. In Iran, nationwide reporting of AFP was established in 1998, and an active surveillance system was later established for non-polio AFP by the Ministry of Health and Medical Education according to WHO guidelines using the same strategy throughout the country. This nationwide health system, which covers all the regions of the country including Khuzestan Province, actively monitors the detection of non-polio AFP. It is mandatory for healthcare 
workers to report and follow all children with AFP. In the health centers to which the cases are reported, a national expert committee evaluates all AFP cases according to standard methods recommended by $\mathrm{WHO}^{20}$, and the data is entered into a WHO standardized form. The demographic data consisted of age, gender, location, vaccination history, month, season and year of involvement, the presence of fever at the beginning of paralysis, symmetry or asymmetry of paralysis, stool test results, and the disease outcome 60 days after the onset for controlling evidence of residual weakness were recorded. All children would undergo diagnostic and therapeutic management. The definitive diagnosis of non-polio cases and poliomyelitis was based on two stool cultures from children with acute flaccid paralysis, and non-polio AFP cases were confirmed with negative cultures.

It was of extreme importance to further categorize the cases by gender and age. Therefore, the cases were divided into three age groups consisting of $0-4,5-9$, and 10-14 years old. Poisson regression was used to estimate the mean annual incidence rate of non-polio AFP per 100,000 individuals under the age of fifteen.

The analyses were performed using SPSS version 22.0 (SPSS Inc., Chicago, IL, USA).
Categorical variables were compared using Pearson's chi-square test. The odds ratio and 95\% confidence interval of each variable were calculated. Statistical significance was set at $\mathrm{p}$ $<0.05$.

\section{Results}

During the eleven-year period from January 2006 to December 2016, a total of 274 AFP cases (an average of 24.9 cases per year) were reported to the center for disease control. One of the WHO targets on AFP surveillance is the detection of at least one AFP case per 100,000 individuals under the age of fifteen as an indicator for measuring the sensitivity of AFP surveillance, which has been met during the previous years.

Cumulatively, the annual non-polio AFP detection rate of Khuzestan Province was 2.04 AFP cases per 100,000 individuals under the age of fifteen between 2006 and 2016. The AFP detection rate in this province increased from 1.46 to 3.11 cases per 100,000 individuals under the age of fifteen in the study period. There were statistically significant fluctuations in annual and monthly occurrences of GBS $(\mathrm{P}=0.003)$. The incidence was low in the initial years of this study, an increasing trend was observed with the number of AFP cases peaking (3.11) in 2010, and then a decline in

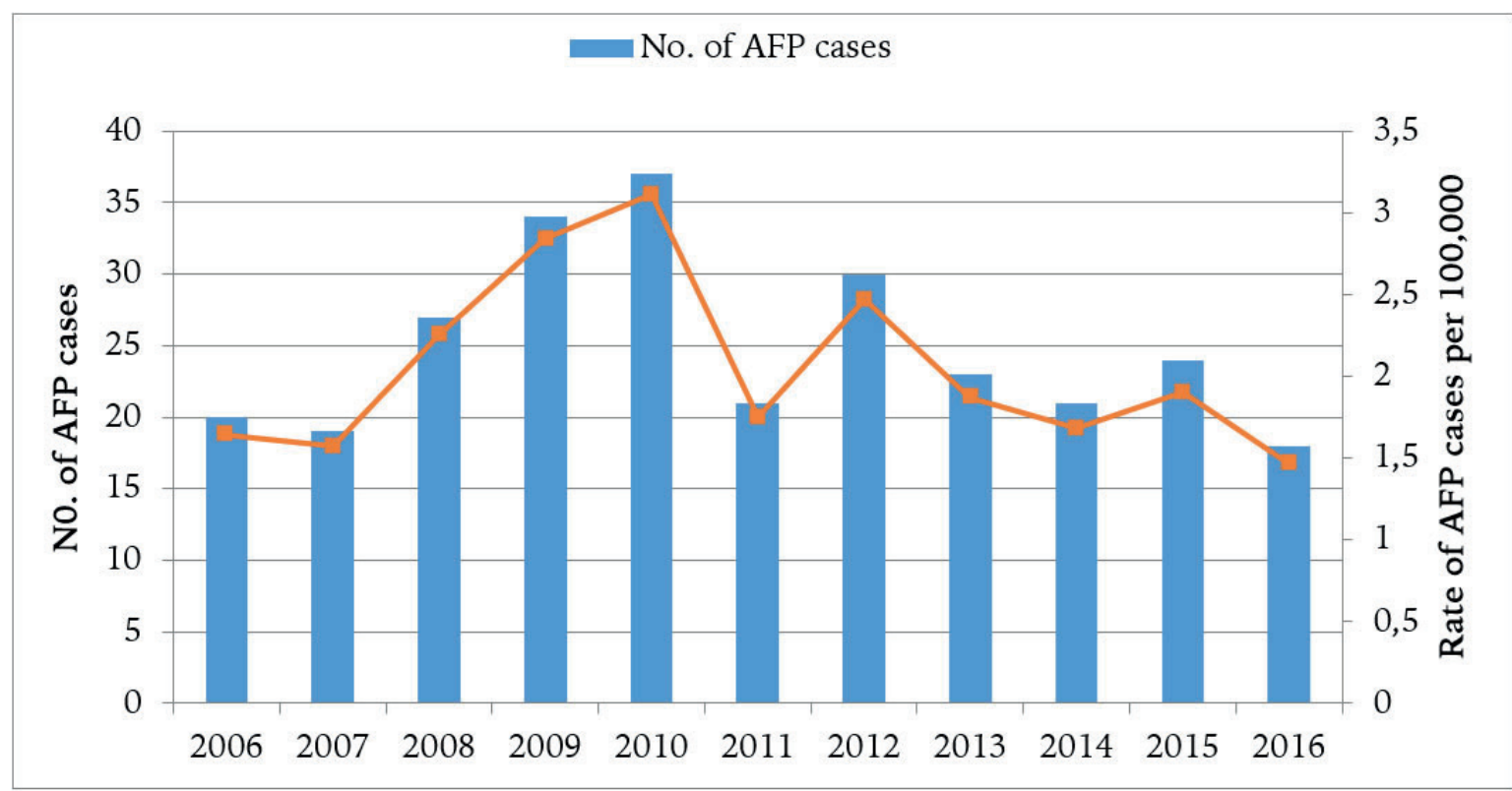

Fig. 1. No. of AFP cases and rate of AFP cases per 100,000 aged less than 15, 2006-2016. 
reported cases was demonstrated from 2011 to 2016 (Fig. 1).

The monthly distribution of AFP cases is shown in Figure 2. The lowest numbers of cumulated cases occurred during July and September with 12 and 14 cases, respectively, while the highest number of cumulated cases was observed in December with 36 cases (Fig. 2).

From 274 cases of AFP, 153 (55.8\%) were males and 121 (44.2\%) were females (male: female ratio 1:0.77). Mean age of patients was $4.97 \pm 3.78$ years. Children aged under five accounted for more than half of the cases

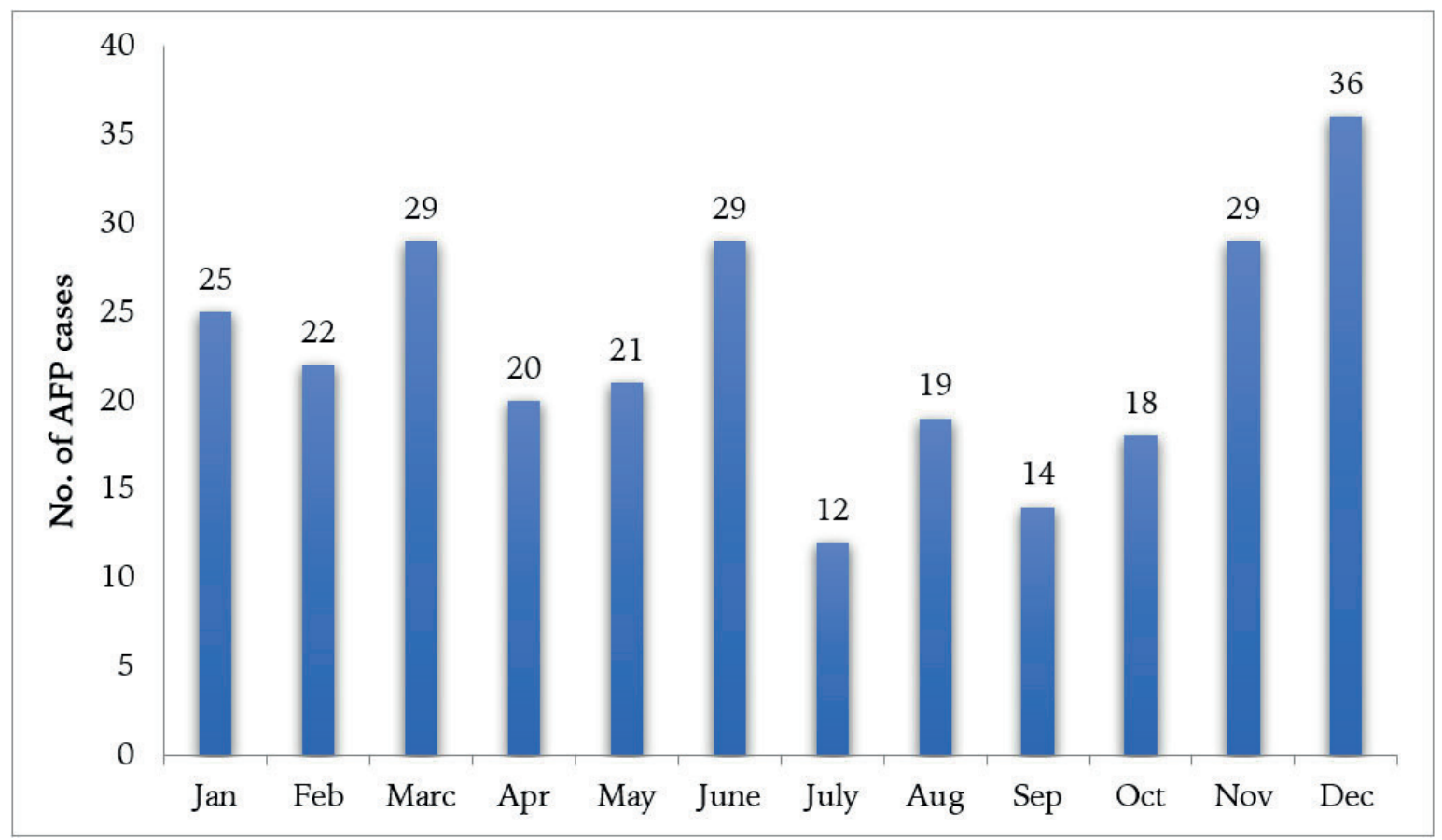

Fig. 2. No. of AFP cases reported by month from 2006 to 2016.

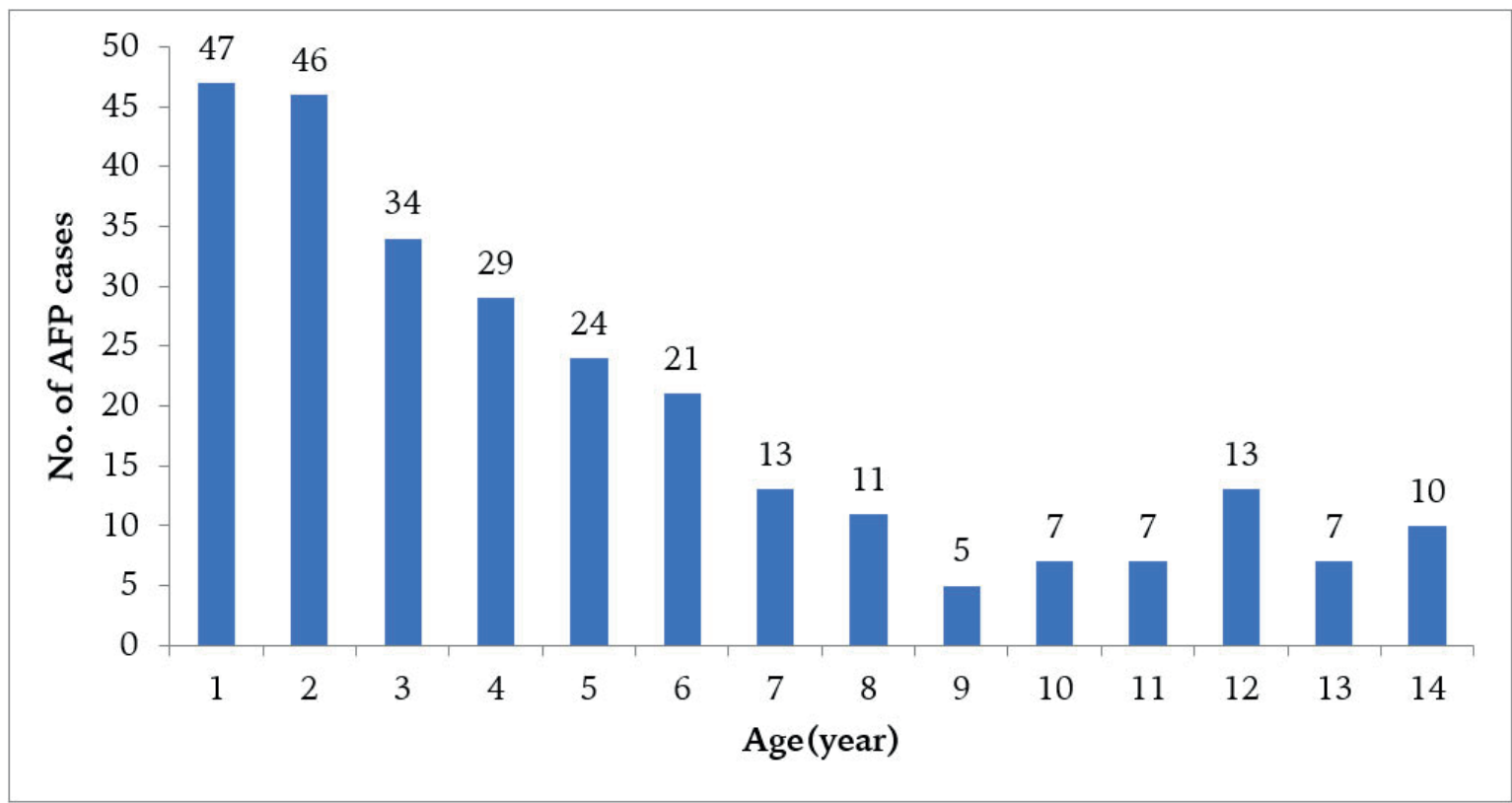

Fig. 3. No. of AFP cases reported by age from 2006 to 2016. 
Table I. Incidence Rate of AFP in Children by Age, Gender and Living Location Using Poisson Regression Analysis.

\begin{tabular}{lccccc}
\hline P value & $95 \%$ CI & Relative risk & $\begin{array}{c}\text { AFP rate per } \\
100,000\end{array}$ & Number (\%) & Variable \\
\hline Sex & & & & & \\
$\quad$ Male & 0.010 & $1.05-1.69$ & 1.33 & 2.22 & $153(55.9)$ \\
$\quad$ Female & - & - & 1.0 & 1.85 & $121(44.2)$ \\
Age (yr) & & & & & \\
$\quad<5$ & 0.0001 & $2.48-4.87$ & 3.47 & 3.29 & $156(56.9)$ \\
$5-9$ & 0.001 & $1.29-2.74$ & 1.88 & 1.86 & $75(27.4)$ \\
$10-14$ & - & - & 1.0 & 0.95 & $43(15.7)$ \\
Living location & & & & & \\
Urban & 0.382 & $0.804-1.37$ & 1.05 & 1.88 & $170(68.5)$ \\
Rural & - & - & 1.0 & 1.79 & $78(31.5)$ \\
Seasons & & & & & $76(27.7)$ \\
Winter & 0.308 & $0.653-1.26$ & 0.907 & 2.27 & $70(25.5)$ \\
Spring & 0.278 & $0.643-1.24$ & 0.893 & 2.09 & $45(16.4)$ \\
Summer & 0.003 & $0.404-0.851$ & 0.587 & 1.34 & $83(30.4)$ \\
Autumn & - & - & 1.0 & 2.47 & \\
\hline
\end{tabular}

$(56.9 \%)$, a majority of cases $(170,68.5 \%)$ resided in urban areas, and 78 cases $(31.5 \%)$ were from rural areas. Most cases were reported in autumn (Table I).

In Table I, the incidence rate of AFP is shown, specified by sex, age, residence, and season. According to Poisson regression analysis, the risk of AFP in children under five years of age was almost twice that of children aged 5-9 and $10-14$ years $(\mathrm{P}=0.001$ and $\mathrm{P}=0.000$, respectively). As shown in Figure 3, the prevalence of AFP gradually decreased with increase in age. There were also statistically significant differences between the risk of AFP among children with gender and season $(\mathrm{P}=$ 0.010 and $P=0.003$, respectively). However, there was no significant difference between residence and risk of AFP $(\mathrm{P}=0.382)$.

The immunization status records showed that $3.9 \%$ of the children had received less than three doses of OPV, $93.3 \%$ had received at least three doses of OPV while the vaccination status of $2.8 \%$ of the children was unknown. Out of 274 AFP cases, 119 (43.5\%) had the fever at the onset of paralysis, and 123 (44.9\%) had asymmetric paralysis.
The stool samples of 274 cases (100\%) were sent for poliovirus isolation. Stool examination was adequate in all cases and was sufficient to exclude poliomyelitis. All stool specimens were tested virologically, but no wild polioviruses were detected. All AFP cases due for 60 days follow- up were examined at 60 days of onset of symptoms.

A variety of diagnoses were identified as the AFP cases, including GBS reported in 204 cases $(74.5 \%)$, followed by myelitis in 8 cases $(2.9 \%)$, arthritis in 7 cases $(2.6 \%)$, poliomyelitis in 4 cases (1.5), infection in 2 cases (0.7), tetanus in 1 case $(0.4)$, and others in 48 cases $(17.5 \%)$. Other conditions included osteomyelitis and acute transient viral synovitis.

\section{Discussion}

The present study represents an effort to better understand the implementation of the regional AFP surveillance system in Khuzestan Province and to report its results from the past eleven years. Furthermore, the estimated incidence of AFP (2.08 cases per 100,000 individuals under the age of 15) is higher than the WHO target of 1 per 100,000 individuals under the age of fifteen $^{15}$, which shows that Khuzestan Province 
has a functional AFP surveillance system that operates relatively well in spite of the challenges it faces other than the geographical region it covers. According to $\mathrm{WHO}$, the estimated AFP incidence rate in Iran has been reported as $3.3 \%$, which demonstrates the success in implementing the AFP surveillance system and suggests major accomplishment in maintaining polio eradication in Iran. ${ }^{8}$ Iran has been certified as a polio-free country by WHO, but there is a high importation risk of wild poliovirus from the endemic neighboring countries of Afghanistan and Pakistan. Hence, there is still a need for robust surveillance for AFP cases to monitor the wild poliovirus circulation and poliomyelitis resurgence.

In this study, we found an increased incidence of AFP in 2009 and 2010, but no precipitating factor could be identified. Ebrahimi et al. ${ }^{21}$ in a similar study in Kerman, a region in central Iran, have also reported the increased incidence of AFP cases in 2009. Increasing incidence of AFP in this period may be caused by an infection, vaccination, and many other factors such as the increase in AFP incidence after vaccination. The findings of a study have shown that AFP incidence has been increased after $\mathrm{N} 1 \mathrm{H} 1$ vaccination. ${ }^{22}$

Our findings indicated that the incidence of AFP was higher among males compared to females, which was consistent with many other published studies ${ }^{23-25}$, indicating that gender does affect the incidence rate of AFP. This can be explained by the fact that there are sex differences in the susceptibility to infectious agents. Generally, males are more prone to develop an infectious disease than females, which may be related to genetic differences in immunologic response. ${ }^{26}$

The age distribution of AFP cases demonstrated that younger age was a risk factor as previously established. ${ }^{6}$ Accordingly, our results showed that $56.3 \%$ of AFP cases occurred in children younger than five years of age. Similarly, previous studies reported a distribution of $66.2 \%$ and $84.8 \%$ among this age group in Iran $^{27}$ and in Nigeria ${ }^{28}$, respectively. These findings indicate that although poliomyelitis can affect any age group, children under the age of five years are more prone to infection. ${ }^{29}$
In this study, the majority of cases were seen during autumn (30.4\%) followed by winter $(27.7 \%)$. Data about the prevalence of AFP in a specific season are inconsistent. The distribution of AFP cases in different seasons differs from one country to another. Generally, in countries with lower temperatures, the AFP cases clustered during the colder seasons of the year, which suggests that different climates might contribute to the high occurrence of AFP in certain seasons in different countries. It is documented that seasonal variation in AFP incidence is attributed to higher rates of infection in certain seasons. ${ }^{6} \mathrm{~A}$ notable point in this study was the significant seasonal variation and a lower incidence of AFP in summer. In Khuzestan, summer is hot, and the mean temperature ranges from $43-52^{\circ} \mathrm{C}$. The severe hot summer temperature may reduce the rate of infectious diseases, which might contribute to the lower occurrence of AFP in this season among children. This finding is consistent with another study in Pakistan by Siddiqi et al..$^{30}$, which found a fewer number of cases during summer.

The present study showed a slight increase in AFP risk in urban centers compared with rural areas. This finding was consistent with another study in Iran by Naeini et al. $^{31}$, which reported most of the AFP cases from urban areas. In another study by Khona et $\mathrm{al}^{32}$, statistically significant differences in the incidence of AFP were reported among urban and rural populations in India. Available data did not provide clear clues to account for this difference in incidence. Given the high frequency of previous infectious diseases in AFP, one might speculate the possibility of a higher concentration of trigger agents that could have affected mainly children living in urban areas, perhaps because of an impaired immunity in children and the higher population density of urban compared to rural areas.

Since the implementation of GPEI program in $1991^{2}$, most AFP cases among all ages worldwide have been attributed to GBS, an immune-mediated condition affecting peripheral nerves and nerve roots. ${ }^{33}$ In this study, GBS was the most common cause of AFP with a frequency of $74.5 \%$, which is consistent with findings from other countries. ${ }^{23,25,34}$ GBS 
has been consistently reported as the most common cause of non-polio AFP in children younger than fifteen years over the last two decades worldwide, with a prevalence ranging from $15 \%$ of cases in Pakistan ${ }^{25}$ to $83.5 \%$ of cases in Iran. ${ }^{31}$ The reason for these variations may be attributed to surveillance sensitivity and possible differential diagnoses, hence an accurate diagnosis of the cause of GBS is important. It is worth mentioning that definite diagnosis of GBS requires access to advanced laboratories and facilities, which is not available in every country.

In this study, no case of poliomyelitis due to wild poliovirus was detected, which is consistent with other findings after January 2001 in Iran. ${ }^{11}$ However, as poliomyelitis is endemic in Pakistan and Afghanistan (two neighboring countries in the eastern border of Iran), as well as frequent travel and immigration from these neighboring countries to Iran, there is potential for spread of the disease; therefore, strict implementation of the surveillance system, especially in provinces and cities with close proximities to the borders, is warranted. The AFP surveillance has been recognized as a gold standard for identifying possible poliomyelitis cases and verifying its eradication. It is therefore essential that international partners and governments continue to support AFP surveillance, both to reliably monitor the successful completion of global polio eradication and to assure that the existing key resources for disease surveillance are not lost but directed towards supporting other critical health programs.

This study had a number of limitations. The application of diagnostic criteria of WHO standard method was conducted retrospectively based on field reports by Iranian medical health care system and the children could not be examined by the researchers. Collecting data and reporting on performance was slightly delayed, and the results are somewhat antiquated.

In this study, like other previous studies performed in Iran, no poliomyelitis case with wild poliovirus was reported, which indicates that the AFP surveillance system in Iran has met most of the WHO-specified epidemiological and laboratory performance standards. The findings of the present study show the improvement in AFP surveillance in the Khuzestan Province, and that the system in place is sensitive enough to pick up any AFP case caused by circulating or imported wild poliovirus. It is also necessary to continually strengthen the AFP surveillance system at the underperforming sub-national levels in order to ensure that the system is robust enough to document the absence of the wild poliovirus in the geographic region, as well as to timely detect any poliovirus re-importation.

This study has ethics approval from the ethics committee of research department of Ahvaz Jundishapur University of Medical Sciences (IR.AJUMS.REC.1395.229).

\section{Acknowledgment}

We would like to thank the health affair deputy of Ahvaz Jundishapur University of Medical Sciences, especially the Acute Flaccid Paralysis committee for providing data regarding children with AFP.

\section{REFERENCES}

1. Razavi SM, Mardani M, Salamati P. Eradication of Polio in the World; Iran is at Risk for Reemerging of Polio: A Review of the Literature. Arch Clin Infect Dis 2016; 11: e36867.

2. Matlin SA, Haslegrave M, Told M, Piper J. The Global Polio Eradication Initiative: Achievements, Challenges and Lessons Learned from 1988-2016. Geneva: Global Health Centre, the Graduate Institute of International and Development Studies. Published February 2017.

3. Derrough T, Salekeen A. Lessons learnt to keep Europe polio-free: A review of outbreaks in the European Union, European Economic Area, and candidate countries, 1973 to 2013. Euro Surveill 2016; 21: 30210

4. Mwengee W, Okeibunor J, Poy A, et al. Polio Eradication Initiative: Contribution to improved communicable diseases surveillance in WHO African region. Vaccine 2016; 34: 5170-5174.

5. Sevencan F, Ertem M, Öner $\mathrm{H}$, et al. Acute flaccid paralysis surveillance in southeastern Turkey, 19992010. Turk J Pediatr 2013; 55: 283-291.

6. Chan DY, Nai-kong L, Yu-lung L, et al. Fifteen years of acute flaccid paralysis surveillance in Hong Kong: Findings from 1997 to 2011. J Paediatr Child Health 2014; 50: 545-552. 
7. Davarpanah MA, Bakhtiari H, Mehrabani D, Khademolhosseini F. A 12-years surveillance of poliomyelitis and acute flaccid paralysis in Fars Province, Southern Iran. Iran Red Crescent Med J 2008; 10: 288-293.

8. Moussavi T, Sadrizadeh B, Zahraei M, Nategh R, Nadim A. Polio eradication in Iran. Arch Iran Med 2012; 15: 107-109.

9. Moradi-Lakeh M, Esteghamati A. National Immunization Program in Iran: whys and why nots. Hum Vaccin Immunother 2013; 9: 112-114.

10. Sadrizadeh B, Zahraei SM. Poliomyelitis eradication in Iran: opportunities and challenges. J Compr Ped 2013; 4: 91-92.

11. Zahraei SM, Sadrizadeh B, Gouya MM. Eradication of poliomyelitis in Iran, a historical perspective. Iran J Public Health. 2009; 38(Suppl 1): 124-126.

12. Polio Eradication Initiative. http://www.emro.who. int/polio/countries/islamic-republic-of-iran.html (Accessed 10.12.2017).

13. Macesic N, Hall V, Mahony A, et al. Acute flaccid paralysis: the new, the old, and the preventable. Open Forum Infect Dis 2016; 3: 1-4.

14. Özalp E, Aydın-Teke T, Tanır G, Özkan M, Bayhan Gí. Transient acute flaccid paralysis and seizures associated with rotavirus gastroenteritis in a child. Turk J Pediatr 2012; 54: 661-663.

15. Tangermann RH, Lamoureux C, Tallis G, Goel A. The critical role of acute flaccid paralysis surveillance in the Global Polio Eradication Initiative. Int Health 2017; 9: 156-163.

16. Desai S, Smith T, Thorley BR, et al. Performance of acute flaccid paralysis surveillance compared with World Health Organization standards. J Paediatr Child Health 2015; 51: 209-214.

17. Sousa IP Jr, Burlandy FM, Oliveira SS, et al. Acute flaccid paralysis laboratorial surveillance in a polio-free country: Brazil, 2005-2014. Hum Vaccin Immunother 2017; 13: 717-723.

18. Morales M. Tangermann RH, Wassilak SG. Progress toward polio eradication-Worldwide, 2015-2016. MMWR Morb Mortal Wkly Rep 2016; 65: 470-473.

19. Statistical Centre of Iran. https://www.amar.org.ir/ english/.(Accessed 9.12.2017).

20. Report of the second meeting of the Global Commission for the Certification of the Eradication of Poliomyelitis Geneva, 1 May 1997 Geneva, World Health Organization, Geneva 1998. http://polioeradication. org/wp-content/uploads/2016/07/9Report.pdf. (Accessed 10.25.2017).

21. Ebrahimi HA, Nakhaee N, Zahmatkesh R, Mirzaee M. Study of acute flaccid paralysis surveillance system in Kerman (Iran) for one decade. Russ Open Med J 2014; 3: 1-4.
22. Lavelle TA, Meltzer MI, Gebremariam A, Lamarand K, Fiore AE, Prosser LA. Community-based values for 2009 pandemic influenza A H1N1 illnesses and vaccination-related adverse events. PloS One 2011; 6: 1-9.

23. Mohsin N, Asimi R. Clinical profile of acute flaccid paralysis: A study from a tertiary care centre in Kashmir, India. Ann Niger Med 2016; 10: 24-29.

24. Poorolajal J, Ghasemi S, Farahani LN, Hosseini AS, Bathaei SJ, Zahiri A. Evaluation of acute flaccid paralysis in hamadan, Iran from 2002 to 2009 Epidemiol Health 2011; 33: 1-5

25. Faheem MU, Haroon MZ, Khan AA, Shaukat M, Anwar SA. Acute Flaccide Paralysis surveillance: A 5 years study of Bannu, Pakistan. J Ayub Med Coll Abbottabad 2015; 27: 673-676.

26. Membo HK, Mweene A, Sadeuh-Mba SA, et al Acute flaccid paralysis surveillance indicators in the Democratic Republic of Congo during 2008-2014. Pan Afr Med J 2016; 24: 1-13.

27. Soltani J, Esmailnasab N, Roshani D, Karimi M, Amjadi MJ. Acute flaccid paralysis and its differential diagnosis in in kurdistan province, Western Iran; an 11-year surveillance. Iran J Pediatr 2014; 24: 131-139.

28. Bassey BE, Rui VG, Gasasira AN, et al. Characteristics of Acute Flaccid Paralysis Reported by the Surveillance System and Verified by WHO Officer in Akwa Ibom State-Nigeria, 2006-2012. Health 2014; 6: 26022610 .

29. Heymann DL. Polio eradication: finishing the job and protecting the investment. Bull World Health Organ 2004; 82: 1 .

30. Siddiqui AI, Bouk GR, Chand L, Mahesar SA, Tunio GM. Variants and variations among gullian barre syndrome presenting as acute flaccid paralysis. PJNS 2014; 9: 12-15.

31. Naeini AE, Ghazavi M, Moghim S, Sabaghi A, Fadaei R. Acute flaccid paralysis surveillance: A 6 years study, Isfahan, Iran. Adv Biomed Res 2015; 4: 99.

32. Khona P, Prayag A, Ashtagi GS. Study of acute flaccid paralysis cases at tertiary care hospital Belagavi, Karnataka. Int J Med Sci Public Health 2017; 6: 398401.

33. Sejvar JJ, Lopez AS, Cortese MM, et al. Acute flaccid myelitis in the United States, August-December 2014: Results of nationwide surveillance. Clin Infect Dis 2016; 63: 737-745.

34. Saraswathy T, Zahrin H, Apandi M, et al. Acute flaccid paralysis surveillance: Looking beyond the global poliomyelitis eradication initiative. Southeast Asian J Trop Med Public Health 2008; 39: 1033-1039. 